\title{
Composição e riqueza florística do componente arbóreo da Floresta Atlântica submontana na região de Imbaú, Município de Silva Jardim, RJ ${ }^{1}$
}

\author{
Fabrício Alvim Carvalho ${ }^{2,3,5}$, Marcelo Trindade Nascimento ${ }^{3}$ e João Marcelo Alvarenga Braga ${ }^{4}$
}

Recebido em 1/9/2005. Aceito em 13/04/2006

\begin{abstract}
RESUMO - (Composição e riqueza florística do componente arbóreo da Floresta Atlântica submontana na região de Imbaú, Município de Silva Jardim, RJ). O presente trabalho teve por objetivo avaliar a composição florística arbórea de um trecho de Floresta Atlântica submontana na região de Imbaú, Silva Jardim, RJ. Esta região formava um contínuo florestal que foi fragmentado há mais de 50 anos por práticas agropecuárias. Cinco fragmentos foram selecionados e em cada um deles foram alocadas quatro parcelas de $100 \times 5 \mathrm{~m}$. Todas as árvores vivas com DAP $\geq 5 \mathrm{~cm}$ foram amostradas e identificadas. No hectare amostrado foram encontradas 161 espécies distribuídas por 39 famílias. A similaridade florística entre os fragmentos foi alta, com índice de Morisita variando de 0,36 a 0,79. As famílias Leguminosae, Lauraceae e Rubiaceae apresentaram a maior riqueza de espécies. A comparação florística com uma floresta madura da região indicou forte decréscimo na riqueza de espécies que, juntamente com a elevada densidade de espécies secundárias iniciais, indicam que estas matas se encontram em estádio sucessional secundário. Analisando a similaridade florística entre a região de Imbaú e outras 17 florestas do Rio de Janeiro, observou-se maior similaridade com as florestas submontanas mais próximas geograficamente. Devido ao avançado processo de fragmentação e à importância ecológica destes fragmentos para a manutenção da flora e fauna, sugere-se que a região de Imbaú deva ser prioritária em programas de conservação e manejo de áreas de Mata Atlântica.
\end{abstract}

Palavras-chave: riqueza florística, Mata Atlântica, fragmentação florestal, conservação, Rio de Janeiro

\begin{abstract}
Floristic composition and richness of the tree community in a submontane Atlantic Forest in the Imbaú region, Silva Jardim, Rio de Janeiro). This study aimed to evaluate tree floristic composition of the submontane Atlantic Forest in the Imbaú region, Silva Jardim, Rio de Janeiro. About 50 years ago, the forest fragmentation process was begun in this region due to farming practices. Five forest remnants were selected and four plots $(100 \times 5 \mathrm{~m})$ were systematically located in each one. All trees with dbh $\geq 5 \mathrm{~cm}$ were sampled and identified. In the one-hectare sample area, 161 species in 39 families were sampled. Species similarity was high between fragments (Morisita index ranging from 0.36 to 0.79 ). The families Leguminosae, Lauraceae and Rubiaceae showed the highest values of species richness. The studied area had a high density of early secondary species and a decrease in canopy species richness when compared to a mature forest nearby. These results indicated characteristics of secondary forests. When compared to 17 forests of Rio de Janeiro State, the Imbaú region showed stronger floristic relationships with submontane forests, especially those geographically closer. Due to its ecological importance for the local flora and fauna, plus the advanced stage of fragmentation in the region, the Imbaú region should be considered as a priority area for adopting conservation and management practices.
\end{abstract}

Key words: floristic richness, Atlantic Forest, forest fragmentation, conservation, Rio de Janeiro

\section{Introdução}

A Floresta Atlântica da costa brasileira é um dos biomas mais ricos em diversidade biológica e mais ameaçados do planeta, considerado um dos cinco mais importantes hotspots de biodiversidade (Myers et al. 2000). No Sudeste brasileiro sua fragmentação já atinge estágio muito avançado e a preservação de suas áreas florestais remanescentes representa um dos maiores problemas de conservação do País (Câmara 1983; Fundação SOS Mata Atlântica 2002; Rambaldi \& Oliveira 2003; Tabarelli et al. 2005).

No Estado do Rio de Janeiro, a Floresta Atlântica encontra-se hoje reduzida a menos de $20 \%$ de sua cobertura original, estando os grandes remanescentes em sua maioria sobre áreas montanhosas inadequadas à agropecuária (Fundação SOS Mata Atlântica 2002). Sua região costeira durante séculos foi alvo de intensas

\footnotetext{
1 Parte da Dissertação de Mestrado do primeiro Autor

2 Universidade de Brasília, Programa de Pós-graduação em Ecologia, Instituto de Ciências Biológicas, C. Postal 04457, 70919-970 Brasília, DF, Brasil

3 Universidade Estadual do Norte Fluminense, Laboratório de Ciências Ambientais (LCA/CBB), Av. Alberto Lamego 2000, Parque Califórnia, 28015-620 Campos dos Goytacazes, RJ, Brasil

4 Instituto de Pesquisas do Jardim Botânico do Rio de Janeiro, Rua Pacheco Leão 915, 22460-030 Rio de Janeiro, RJ, Brasil

5 Autor para correspondência: fabricioalvim@yahoo.com.br
} 
perturbações antrópicas, intensificadas nas últimas sete décadas através da extração madeireira, caça ou da substituição de suas florestas por áreas agrícolas e pelo processo de urbanização desordenada (Dean 1996). A paisagem atual desta região encontra-se bastante fragmentada e desconectada, representada em sua quase totalidade por pequenas manchas florestais, isoladas e impactadas, circundadas por extensas matrizes antrópicas como pastos, monoculturas e áreas de desenvolvimento urbano (Fundação SOS Mata Atlântica 2002).

O Município de Silva Jardim, localizado na região Centro-Norte do Estado do Rio de Janeiro, ainda preserva mais de $30 \%$ (cerca de $340 \mathrm{~km}^{2}$ ) de sua cobertura florestal original (Fundação SOS Mata Atlântica 2002). Seus remanescentes encontram-se totalmente inseridos no domínio da Floresta Ombrófila Densa (Veloso et al. 1991), e parte de seu território integra ainda o corredor de biodiversidade da Serra do Mar (Ayres et al. 2005). O apelo para a preservação de sua cobertura florestal, através do uso do micoleão-dourado (Leontopithecus rosalia) como espécie bandeira, resultou na criação da primeira Reserva Biológica brasileira, a Reserva Biológica de Poço das Antas, em 1974 (IBDF 1981). Passados 30 anos, o município de Silva Jardim destaca-se no cenário nacional também por ser a região com o maior número de Unidades de Conservação do Brasil, com mais de uma dezena de Reservas Particulares do Patrimônio Natural (RPPN) implementadas (Carvalho et al. 2004).

A Floresta Atlântica desta região vem sendo estudada em termos florísticos e fitossociológicos, através dos trabalhos de Borém \& Oliveira Filho (2002), F.A. Carvalho (dados não publicados), G.M.S. Neves (dados não publicados), R.R. Guedes-Bruni (dados não publicados) e S.V.A. Pessoa (dados não publicados). Entretanto, a maior parte destes estudos foi realizada dentro da Reserva Biológica de Poço das Antas (G.M.S. Neves, dados não publicados; R.R. Guedes-Bruni, dados não publicados; S.V.A. Pessoa, dados não publicados). Para diversos remanescentes, principalmente aqueles localizados em propriedades particulares e mais susceptíveis aos impactos antrópicos, ainda não há informações sobre suas comunidades vegetais. Nesta situação está a região de Imbaú, que ainda preserva diversos remanescentes de Floresta Ombrófila Densa Submontana, importantes como habitats de micos-leões-dourado reintroduzidos e silvestres (Carvalho et al. 2004).

Ressalta-se que o estudo promovido pelo Ministério do Meio Ambiente (MMA 2000) para a identificação de ações e áreas prioritárias para a conservação da biodiversidade da Floresta Atlântica considerou os remanescentes dessa região de extrema importância ecológica para a conservação da fauna e flora. Dada a representatividade, o alto grau de fragmentação e a pressão antrópica ao qual estão sujeitos, recomendou-se ainda que a região seja manejada de forma a constituir um corredor ecológico. Para tal feito, informações sobre a composição florística destes remanescentes são necessárias.

Neste contexto, o presente trabalho teve por objetivos avaliar a composição florística e a riqueza do componente arbóreo da Floresta Atlântica submontana da região de Imbaú, no Município de Silva Jardim, e comparar os dados obtidos com os de outros trechos de Floresta Atlântica do Estado do Rio de Janeiro. Desta forma, busca-se disponibilizar informações que auxiliem nas tomadas de decisões para futuras ações de manejo, recuperação e conservação da vegetação e, conseqüentemente, da diversidade biológica desta região.

\section{Material e métodos}

Área de estudo - A região de Imbaú está localizada no Município de Silva Jardim, RJ (42²8'02'”W e $22^{\circ} 37^{\prime} 01$ 'S), e pertence à Área de Proteção Ambiental da Bacia do Rio São João/Mico-Leão-Dourado/ IBAMA (Primo \& Völker 2003). Importante por ser área endêmica de ocorrência do mico-leão-dourado (Leontopithecus rosalia), a região de Imbaú ainda preserva remanescentes de Floresta Atlântica, porém fragmentados e de tamanhos reduzidos, com poucos excedendo 1.000 ha (Fundação SOS Mata Atlântica 2002). Os solos são classificados como Latossolo Amarelo Distrófico (IBGE 2004), com exceção do fragmento EST que apresenta solo hidromórfico (Gleissolo) (J. Lima \& D.M. Villela, dados não publicados). A vegetação predominante é a Floresta Ombrófila Densa Submontana (Veloso et al. 1991), em diferentes estágios sucessionais (Programa Mata Atlântica 1996). O clima da região é o tropical úmido com estação chuvosa no verão e sem inverno pronunciado, estando enquadrado pela classificação de Koeppen (1948) no tipo As (tropical chuvoso com estação seca no inverno). A precipitação anual oscila entre 1.500 e $2.000 \mathrm{~mm}$, sendo os meses de novembro a março os mais chuvosos e de maiores temperaturas (Primo \& Völker 2003).

A região de Imbaú formava um maciço florestal contínuo, fragmentado a partir do início do século 
passado para a implantação de agropecuária extensiva. Nesta região, cinco trechos florestais resultantes da fragmentação foram selecionados para a realização deste estudo: "Fazenda Afetiva-Jorge" (AFT - 19 ha), "Fazenda Estreito" (EST - 21 ha), "Fazenda Imbaú" (IMB - 130 ha), "Fazenda Andorinhas" (AND - 145 ha) e "Sítio do Professor" (STP - 155 ha). Estes fragmentos encontram-se isolados há pelo menos 50 anos, situam-se próximos uns dos outros com distância máxima de cerca de $2 \mathrm{~km}$ (Fig. 1) e estão imersos em uma matriz antrópica composta quase que exclusivamente por pastagens, além de pequenas culturas de subsistência (frutas cítricas e coco) e algumas áreas de regeneração natural (capoeiras). Optou-se pelo estudo destes fragmentos por se tratarem de áreas utilizadas no programa conservação do micoleão-dourado (Carvalho et al. 2004; Ruiz-Miranda et al. 2004), e que carecem de informações a respeito de suas comunidades vegetais.

Análise florística - Para a análise da vegetação utilizouse o método de parcelas longitudinais visando à obtenção de uma maior heterogeneidade florística, diminuindo assim as chances da amostragem permanecer restrita a uma mancha vegetal (Causton

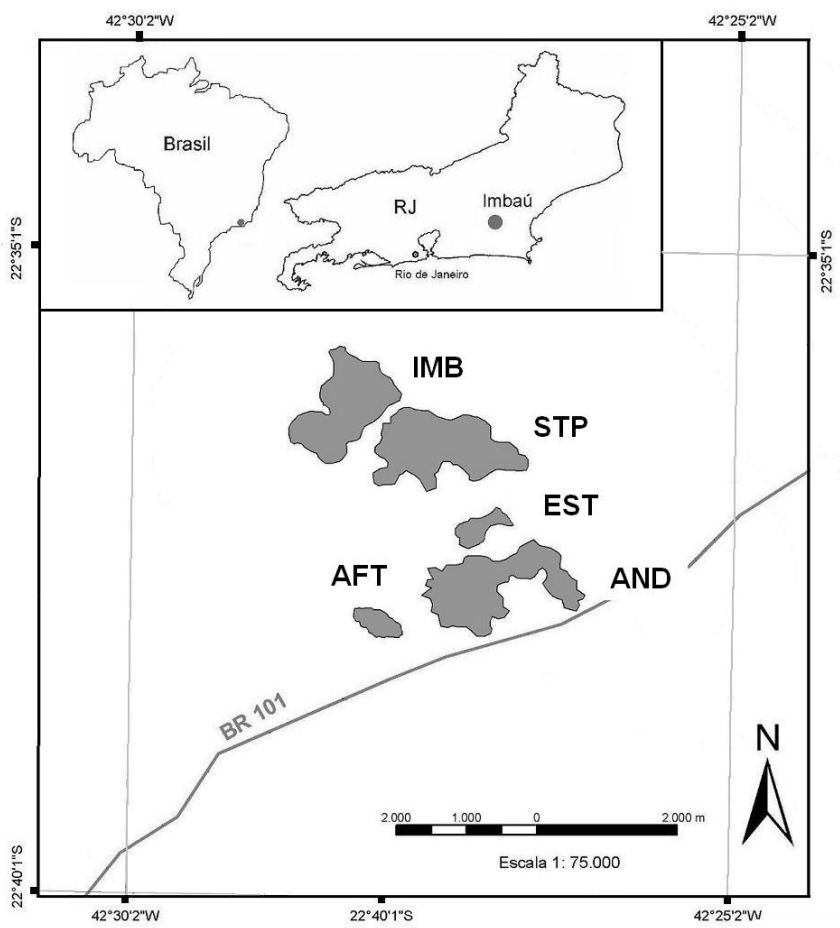

Figura 1. Localização geográfica dos fragmentos de Floresta Ombrófila Densa Submontana estudados na região de Imbaú, Município de Silva Jardim, RJ. AFT - Faz. Afetiva (19 ha); AND - Faz. Andorinhas (145 ha); EST - Faz. Estreito (21 ha); IMB - Faz. Imbaú (130 ha); STP - Sítio do Professor (155 ha).
1988). Ao longo de cada fragmento foram alocadas sistematicamente quatro parcelas de $100 \times 5 \mathrm{~m}$ no sentido norte-sul, cada uma distando pelo menos $100 \mathrm{~m}$ da outra e $20 \mathrm{~m}$ das bordas, correspondendo a uma área amostral de 0,2 ha em cada fragmento e área total amostrada de 1,0 ha.

Dentro das parcelas, todas as árvores vivas (com exceção de lianas) com DAP (diâmetro à altura do peito $=1,30 \mathrm{~m}$ do solo) maior ou igual a $5,0 \mathrm{~cm}$ foram marcadas com plaquetas de alumínio numeradas, e amostras de material botânico, fértil e ou vegetativo, foram coletadas para o auxílio nas identificações taxonômicas. A identificação botânica foi realizada através de consultas ao Herbário do Instituto de Pesquisas do Jardim Botânico do Rio de Janeiro (RB), bibliografia especializada e consultas a especialistas. O material coletado encontra-se depositado no Herbário do Centro de Biociências \& Biotecnologia da Universidade Estadual do Norte Fluminense (HUENF) e duplicatas foram enviadas para o Herbário RB. Foi adotada a classificação de famílias segundo Cronquist (1981), exceto Leguminosae (Fabaceae) s. lat.) que foi subdividida em três subfamílias (Caesalpinioideae, Mimosoideae e Papilionoideae). As espécies tiveram seus binômios confirmados e atualizados através do "software" do índice de espécies do Royal Botanical Garden of Kew (1993), ou por meio de literatura especializada.

As espécies foram classificadas em grupos ecológicos segundo suas características sucessionais e síndrome de dispersão. Esta classificação foi baseada em diversos autores que se utilizaram destes dados nos seus estudos, abrangendo trabalhos em florestas do domínio da Floresta Atlântica. As espécies foram classificadas segundo suas características sucessionais em pioneiras ( $\mathrm{Pi}$ ), secundárias iniciais ( $\mathrm{Si}$ ) e secundárias tardias (St), segundo proposto por Gandolfi et al. (1995). Para as síndromes de dispersão adotou-se a terminologia de Van der Pijl (1982), sendo as espécies classificadas em zoocóricas, anemocóricas e autocóricas.

A similaridade de espécies entre os cinco fragmentos foi estimada através do índice de similaridade quantitativo (densidade) de Morisita (mod. Horn) (Brower \& Zar 1984). Para o cálculo da similaridade de espécies entre a região de Imbaú e outros trechos de Floresta Atlântica do Estado do Rio de Janeiro foram compilados os dados dos inventários florísticos de 17 remanescentes, nos quais apenas os indivíduos com DAP $\geq 5 \mathrm{~cm}$ identificados no nível específico foram incluídos na análise. A partir desta 
compilação, foi elaborada uma matriz de dados binários (presença/ausência) das espécies para o cálculo do índice de similaridade de Jaccard (Brower \& Zar 1984). Este índice foi adotado por possibilitar a comparação de floras gerais de áreas maiores, uma vez que leva em consideração apenas a presença da espécie (Felfili \& Rezende 2003).

\section{Resultados e discussão}

No hectare amostrado na região de Imbaú foram registrados 1591 indivíduos distribuídos em 161 morfoespécies, 107 gêneros e 42 famílias (Tab. 1). Das morfo-espécies, 143 (88,8\% do total) foram identificadas no nível específico, $11(6,8 \%)$ no nível de

Tabela 1. Famílias e espécies amostradas nos fragmentos de Floresta Ombrófila Densa Submontana da região de Imbaú, Silva Jardim, RJ, e classificação por grupos ecológicos. As espécies estão organizadas por ordem alfabética das famílias botânicas. Fragmentos de ocorrência: Faz. Afetiva-Jorge (AFT), Faz. Estreito (EST), Faz. Imbaú (IMB), Faz. Andorinhas (AND) e Sítio do Professor (STP). Grupos ecológicos: Classificação sucessional (CS) (sensu Gandolfi et al. 1995): pioneira (Pi), secundária inicial (Si), secundária tardia (St) e não classificada (Nc). Síndrome de dispersão (SD) (sensu van der Pij1 1982): anemocórica (Amem), autocórica (Autoc) e zoocórica (Zooc). *Espécies exóticas.

\begin{tabular}{|c|c|c|c|c|c|c|c|}
\hline \multirow[b]{2}{*}{ Família/Espécie } & \multicolumn{2}{|c|}{ Grupos ecológicos } & \multicolumn{5}{|c|}{ Fragmentos de ocorrência } \\
\hline & CS & SD & AFT & EST & IMB & AND & STP \\
\hline \multicolumn{8}{|l|}{ ANACARDIACEAE } \\
\hline Astronium graveolens Jacq. & $\mathrm{Si}$ & Anem & & $\mathrm{X}$ & & $\mathrm{X}$ & \\
\hline Tapirira guianensis Aubl. & $\mathrm{Si}$ & Zooc & $\mathrm{X}$ & & & $\mathrm{X}$ & \\
\hline \multicolumn{8}{|l|}{ ANNONACEAE } \\
\hline Guatteria australis A. St.-Hil. & $\mathrm{Si}$ & Zooc & $\mathrm{X}$ & $\mathrm{X}$ & & & \\
\hline G. candolleana Schlecht. & $\mathrm{Si}$ & Zooc & $\mathrm{X}$ & $\mathrm{X}$ & $\mathrm{X}$ & $\mathrm{X}$ & \\
\hline G. xylopioides R.E. Fr. & $\mathrm{Si}$ & Zooc & & $\mathrm{X}$ & & & \\
\hline Rollinia dolabripetala (Raddi) R.E. Fr. & $\mathrm{Si}$ & Zooc & & $\mathrm{X}$ & $\mathrm{X}$ & $\mathrm{X}$ & \\
\hline Xylopia sericea A. St.-Hil. & $\mathrm{Pi}$ & Zooc & $\mathrm{X}$ & $\mathrm{X}$ & $\mathrm{X}$ & $\mathrm{X}$ & $\mathrm{X}$ \\
\hline \multicolumn{8}{|l|}{ APOCYNACEAE } \\
\hline Himatanthus lancifolius (Müll. Arg.) Woodson & St & Anem & $\mathrm{X}$ & & & $\mathrm{X}$ & \\
\hline Rauvolfia grandiflora Mart. ex A. DC. & St & Zooc & & & & $\mathrm{X}$ & \\
\hline Tabernaemontana catharinensis A.DC. & $\mathrm{Si}$ & Anem & $\mathrm{X}$ & & & $\mathrm{X}$ & $\mathrm{X}$ \\
\hline T. laeta Mart. & St & Anem & & & & & $\mathrm{X}$ \\
\hline \multicolumn{8}{|l|}{ ARECACEAE } \\
\hline Astrocaryum aculeatissimum (Schott) Burret & $\mathrm{Pi}$ & Zooc & $\mathrm{X}$ & & & & $\mathrm{X}$ \\
\hline Euterpe edulis Mart. & St & Zooc & $\mathrm{X}$ & & & $\mathrm{X}$ & $\mathrm{X}$ \\
\hline \multicolumn{8}{|l|}{ ASTERACEAE } \\
\hline Vernonanthura discolor (Less.) H. Rob. & $\mathrm{Pi}$ & Anem & $\mathrm{X}$ & & $\mathrm{X}$ & & \\
\hline \multicolumn{8}{|l|}{ BIGNONIACEAE } \\
\hline Adenocalymma subsessilifolium DC. & $\mathrm{Si}$ & Anem & & & & $\mathrm{X}$ & $\mathrm{X}$ \\
\hline Cybistax antisyphilitica (Mart.) Mart. & $\mathrm{Si}$ & Anem & & & $\mathrm{X}$ & $\mathrm{X}$ & \\
\hline Jacaranda bracteata Bureau \& K. Schum. & $\mathrm{Si}$ & Anem & $\mathrm{X}$ & & & & \\
\hline J. macrantha Cham. & $\mathrm{Si}$ & Anem & & & & $\mathrm{X}$ & \\
\hline J. puberula Cham. & $\mathrm{Si}$ & Anem & & & & $\mathrm{X}$ & \\
\hline Sparattosperma leucanthum (Vel1.) K. Schum. & $\mathrm{Pi}$ & Anem & $\mathrm{X}$ & & $\mathrm{X}$ & $\mathrm{X}$ & $\mathrm{X}$ \\
\hline Tabebuia chrysotricha (Mart. ex DC.) Standl. & St & Anem & $\mathrm{X}$ & & & & \\
\hline T. heptaphylla (Vell.) Toledo & St & Anem & $\mathrm{X}$ & & $\mathrm{X}$ & $\mathrm{X}$ & $\mathrm{X}$ \\
\hline T. serratifolia (Vahl) Nichols. & St & Anem & & & & $\mathrm{X}$ & \\
\hline \multicolumn{8}{|l|}{ BOMBACACEAE } \\
\hline Ceiba crispiflora (HBK) Ravenna & St & Anem & & & & & $\mathrm{X}$ \\
\hline Pseudobombax grandiflorum (Cav.) A. Robyns & St & Anem & & & & & $\mathrm{X}$ \\
\hline \multicolumn{8}{|l|}{ BORAGINACEAE } \\
\hline Cordia sellowiana Cham. & $\mathrm{Si}$ & Zooc & $\mathrm{X}$ & & & & \\
\hline C. trichoclada DC. & $\mathrm{Si}$ & Zooc & $\mathrm{X}$ & & & $\mathrm{X}$ & \\
\hline \multicolumn{8}{|l|}{ BURSERACEAE } \\
\hline Protium heptaphyllum (Aubl.) March. & $\mathrm{Si}$ & Zooc & & $\mathrm{X}$ & $\mathrm{X}$ & & \\
\hline \multicolumn{8}{|l|}{ CARICACEAE } \\
\hline Jacaratia heptaphylla (Vell.) A. DC. & St & Zooc & & & $\mathrm{X}$ & & \\
\hline
\end{tabular}


Tabela 1 (continuação)

\begin{tabular}{|c|c|c|c|c|c|c|c|}
\hline \multirow[b]{2}{*}{ Família/Espécie } & \multicolumn{2}{|c|}{ Grupos ecológicos } & \multicolumn{5}{|c|}{ Fragmentos de ocorrência } \\
\hline & $\mathrm{CS}$ & $\mathrm{SD}$ & AFT & EST & IMB & AND & STP \\
\hline \multicolumn{8}{|l|}{ CECROPIACEAE } \\
\hline Cecropia glaziovii Snethl. & $\mathrm{Pi}$ & Zooc & & $\mathrm{X}$ & & & \\
\hline C. hololeuca Miq. & $\mathrm{Pi}$ & Zooc & & $\mathrm{X}$ & $\mathrm{X}$ & $\mathrm{X}$ & \\
\hline \multicolumn{8}{|l|}{ CELASTRACEAE } \\
\hline Maytenus longifolia Reiss. ex Loes. & St & Zooc & & & & $\mathrm{X}$ & $\mathrm{X}$ \\
\hline \multicolumn{8}{|l|}{ CHRYSOBALANACEAE } \\
\hline Chrysobalanaceae sp. 1 & St & Zooc & & & & & $\mathrm{X}$ \\
\hline Chrysobalanaceae sp. 2 & St & Zooc & & & & & $\mathrm{X}$ \\
\hline \multicolumn{8}{|l|}{ CLUSIACEAE } \\
\hline Tovomitopsis paniculata Planch. \& Triana & $\mathrm{Nc}$ & Zooc & $\mathrm{X}$ & & & & \\
\hline Vismia guianensis (Aubl.) Choisy & $\mathrm{Si}$ & Zooc & & & & $\mathrm{X}$ & \\
\hline \multicolumn{8}{|l|}{ ELAEOCARPACEAE } \\
\hline Sloanea garckeana Mart. & St & Zooc & & & & $\mathrm{X}$ & \\
\hline \multicolumn{8}{|l|}{ ERYTHROXYLACEAE } \\
\hline Erythroxylum pulchrum A. St.-Hil. & St & Zooc & & & & $\mathrm{X}$ & \\
\hline \multicolumn{8}{|l|}{ EUPHORBIACEAE } \\
\hline Actinostemon verticillatus (Klotzsch) Baill. & $\mathrm{Si}$ & Auto & & & & $\mathrm{X}$ & \\
\hline Alchornea glandulosa Poepp. & $\mathrm{Si}$ & Zooc & & $\mathrm{X}$ & $\mathrm{X}$ & $\mathrm{X}$ & $\mathrm{X}$ \\
\hline A. triplinervia (Spreng.) Müll. Arg. & $\mathrm{Si}$ & Zooc & $\mathrm{X}$ & & $\mathrm{X}$ & & $\mathrm{X}$ \\
\hline Aparisthmium cordatum (A. Juss.) Baill. & $\mathrm{Si}$ & Zooc & $\mathrm{X}$ & & & & \\
\hline Hieronyma alchorneoides Fr. Allem. & $\mathrm{Si}$ & Zooc & $\mathrm{X}$ & & & $\mathrm{X}$ & \\
\hline Mabea fistulifera Mart. & $\mathrm{Pi}$ & Zooc & & & & & $\mathrm{X}$ \\
\hline Pera glabrata (Schoot) Poepp. ex Baill. & $\mathrm{Si}$ & Zooc & $\mathrm{X}$ & & & & \\
\hline P. leandrii Baill. & St & Zooc & $\mathrm{X}$ & & & & \\
\hline Sapium glandulatum (Vell.) Pax & $\mathrm{Si}$ & Zooc & & & $\mathrm{X}$ & & \\
\hline \multicolumn{8}{|l|}{ FLACOURTIACEAE } \\
\hline Casearia arborea (Rich.) Urb. & $\mathrm{Si}$ & Zooc & & & $\mathrm{X}$ & $\mathrm{X}$ & $\mathrm{X}$ \\
\hline C. sylvestris $\mathrm{Sw}$ & $\mathrm{Si}$ & Zooc & $\mathrm{X}$ & $\mathrm{X}$ & $\mathrm{X}$ & $\mathrm{X}$ & $\mathrm{X}$ \\
\hline \multicolumn{8}{|l|}{ ICACINACEAE } \\
\hline Leretia cordata Vell. & St & Zooc & $\mathrm{X}$ & & & & \\
\hline \multicolumn{8}{|l|}{ LACISTEMACEAE } \\
\hline Lacistema pubescens Mart. & $\mathrm{Si}$ & Zooc & $\mathrm{X}$ & $\mathrm{X}$ & $\mathrm{X}$ & $\mathrm{X}$ & $\mathrm{X}$ \\
\hline \multicolumn{8}{|l|}{ LAURACEAE } \\
\hline Aiouea saligna Meisn. & St & Zooc & & & $\mathrm{X}$ & $\mathrm{X}$ & \\
\hline Endlicheria paniculata (Spreng.) J.F. Macbr. & St & Zooc & & $\mathrm{X}$ & & & \\
\hline Licaria armenica (Nees) Kosterm. & St & Zooc & & & & $\mathrm{X}$ & \\
\hline Nectandra megapotamica (Spreng.) Mez & St & Zooc & & & $\mathrm{X}$ & $\mathrm{X}$ & \\
\hline N. membranaceae (SW.) Griseb. & St & Zooc & & & $\mathrm{X}$ & & $\mathrm{X}$ \\
\hline N. oppositifolia Nees. & $\mathrm{Si}$ & Zooc & $\mathrm{X}$ & $\mathrm{X}$ & $\mathrm{X}$ & $\mathrm{X}$ & $\mathrm{X}$ \\
\hline N. puberula (Schott) Nees & $\mathrm{Si}$ & Zooc & $\mathrm{X}$ & $\mathrm{X}$ & $\mathrm{X}$ & $\mathrm{X}$ & $\mathrm{X}$ \\
\hline Ocotea aciphylla (Nees) Mez & St & Zooc & & & $X$ & & \\
\hline O. aniboides $\mathrm{Mez}$ & St & Zooc & $\mathrm{X}$ & $\mathrm{X}$ & & $\mathrm{X}$ & \\
\hline O. diospyrifolia (Meisn.) Mez & St & Zooc & & & $\mathrm{X}$ & $\mathrm{X}$ & $\mathrm{X}$ \\
\hline O. elegans $\mathrm{Mez}$ & St & Zooc & $\mathrm{X}$ & & & & \\
\hline O. laxa (Nees) Mez & St & Zooc & & $\mathrm{X}$ & & & \\
\hline O. odorifera (Vell.) Rohwer & St & Zooc & & & $\mathrm{X}$ & & $\mathrm{X}$ \\
\hline Persea americana Mill.* & $\mathrm{Nc}$ & Zooc & $\mathrm{X}$ & & & $\mathrm{X}$ & \\
\hline \multicolumn{8}{|l|}{ LECYTHIDACEAE } \\
\hline Cariniana legalis (Mart.) Kuntze & St & Anem & & & & & $\mathrm{X}$ \\
\hline Cariniana sp. 1 & St & Anem & & & & $\mathrm{X}$ & \\
\hline Lecythis sp. 1 & St & Zooc & & & & $\mathrm{X}$ & \\
\hline \multicolumn{8}{|l|}{ LEGUMINOSAE - Caesalpinioideae } \\
\hline Apuleia leiocarpa Vog. & $\mathrm{Si}$ & Anem & $\mathrm{X}$ & & & $\mathrm{X}$ & $\mathrm{X}$ \\
\hline Bauhinia forficata Link & $\mathrm{Si}$ & Auto & & & $\mathrm{X}$ & & $\mathrm{X}$ \\
\hline Chamaecrista ensiformis (Vell.) Irwin \& Barneby & $\mathrm{Si}$ & Auto & $\mathrm{X}$ & $\mathrm{X}$ & & $\mathrm{X}$ & \\
\hline Exostyles venusta Schott ex Spreng. & St & Zooc & & & & $\mathrm{X}$ & \\
\hline
\end{tabular}


Tabela 1 (continuação)

\begin{tabular}{|c|c|c|c|c|c|c|c|}
\hline \multirow[b]{2}{*}{ Família/Espécie } & \multicolumn{2}{|c|}{ Grupos ecológicos } & \multicolumn{5}{|c|}{ Fragmentos de ocorrência } \\
\hline & $\mathrm{CS}$ & SD & AFT & EST & IMB & AND & STP \\
\hline \multicolumn{8}{|l|}{ LEGUMINOSAE - Caesalpinioideae } \\
\hline Melanoxylon brauna Schott & St & Anem & & & & & $\mathrm{X}$ \\
\hline Peltogyne angustiflora Ducke & St & Anem & & & $\mathrm{X}$ & & \\
\hline \multicolumn{8}{|l|}{ LEGUMINOSAE - Mimosoideae } \\
\hline Acacia polyphylla Benth. & $\mathrm{Si}$ & Anem & & $\mathrm{X}$ & $\mathrm{X}$ & & \\
\hline Albizia polycephalla (Benth.) Killip ex Record & $\mathrm{Si}$ & Anem & & & $\mathrm{X}$ & $\mathrm{X}$ & \\
\hline Inga edulis Mart. & $\mathrm{Pi}$ & Zooc & & $\mathrm{X}$ & & & \\
\hline Mimosa bimucronata (DC.) Kuntze & $\mathrm{Pi}$ & Anem & & $\mathrm{X}$ & $\mathrm{X}$ & & \\
\hline Plathymenia foliolosa Benth. & St & Anem & $\mathrm{X}$ & $\mathrm{X}$ & $\mathrm{X}$ & $\mathrm{X}$ & $\mathrm{X}$ \\
\hline Pseudopiptadenia contorta (DC.) G.P. Lewis \& M.P. Lima & $\mathrm{Pi}$ & Anem & $\mathrm{X}$ & & $\mathrm{X}$ & & \\
\hline \multicolumn{8}{|l|}{ LEGUMINOSAE - Papilionoideae } \\
\hline Andira anthelmia (Vell.) J.F. Macbr. & St & Zooc & & & & $\mathrm{X}$ & \\
\hline Dalbergia nigra (Vell.) Fr. Allem. ex Benth. & St & Anem & $\mathrm{X}$ & & & & $\mathrm{X}$ \\
\hline Lonchocarpus cultratus (Vell.) A.M.G. Azeredo \& H. Lima & St & Anem & & & $\mathrm{X}$ & & \\
\hline Machaerium brasiliensis Vog. & $\mathrm{Si}$ & Anem & $\mathrm{X}$ & & $\mathrm{X}$ & $\mathrm{X}$ & \\
\hline M. nictitans (Vell.) Benth. & St & Anem & & $\mathrm{X}$ & & & \\
\hline Myrocarpus frondosus Fr. Allem. & St & Anem & & & & & $\mathrm{X}$ \\
\hline Platycyanus regnellii Benth. & St & Anem & & $\mathrm{X}$ & $\mathrm{X}$ & & $\mathrm{X}$ \\
\hline Platymiscium floribudum Vog. & St & Anem & & $\mathrm{X}$ & & & \\
\hline Platypodium elegans Vog. & St & Anem & $\mathrm{X}$ & & & & \\
\hline Swartzia simplex (Sw.) Spreng. & St & Zooc & $\mathrm{X}$ & & & & \\
\hline \multicolumn{8}{|l|}{ MELASTOMATACEAE } \\
\hline Miconia cinnamomifolia (DC.) Naudin & $\mathrm{Pi}$ & Zooc & $\mathrm{X}$ & $\mathrm{X}$ & $\mathrm{X}$ & $\mathrm{X}$ & \\
\hline M. holosericea (L.) DC. & $\mathrm{Pi}$ & Zooc & & $\mathrm{X}$ & & & \\
\hline M. hypoleuca (Benth.) Triana & $\mathrm{Pi}$ & Zooc & & & & & $\mathrm{X}$ \\
\hline M. prasina $(\mathrm{Sw}.) \mathrm{DC}$ & $\mathrm{Pi}$ & Zooc & & $\mathrm{X}$ & & & \\
\hline Tibouchina granulosa Cogn. & $\mathrm{Pi}$ & Zooc & & & $\mathrm{X}$ & & \\
\hline \multicolumn{8}{|l|}{ MELIACEAE } \\
\hline Cabralea canjerana (Vell.) Mart. & $\mathrm{Si}$ & Zooc & $\mathrm{X}$ & $\mathrm{X}$ & $\mathrm{X}$ & $\mathrm{X}$ & \\
\hline Cedrela fissilis Vell. & St & Zooc & & $\mathrm{X}$ & & & \\
\hline Guarea guidonia (L.) Sleumer & $\mathrm{Si}$ & Zooc & $\mathrm{X}$ & $\mathrm{X}$ & $\mathrm{X}$ & $\mathrm{X}$ & $X$ \\
\hline G. macrophylla Vahl & St & Zooc & & & $\mathrm{X}$ & $\mathrm{X}$ & $\mathrm{X}$ \\
\hline Trichilia elegans A. Juss. & St & Zooc & & $\mathrm{X}$ & & & \\
\hline T. lepidota Mart. & St & Zooc & & $\mathrm{X}$ & & & \\
\hline \multicolumn{8}{|l|}{ MONIMIACEAE } \\
\hline Siparuna guianensis (Tul.) A.DC. & $\mathrm{Si}$ & Zooc & $\mathrm{X}$ & $\mathrm{X}$ & $\mathrm{X}$ & $\mathrm{X}$ & $\mathrm{X}$ \\
\hline Siparuna sp. 1 & $\mathrm{Si}$ & Zooc & & & $\mathrm{X}$ & $\mathrm{X}$ & \\
\hline \multicolumn{8}{|l|}{ MORACEAE } \\
\hline Brosimum guianense (Aubl.) Huber ex Ducke & $\mathrm{Si}$ & Zooc & $\mathrm{X}$ & & & $\mathrm{X}$ & $\mathrm{X}$ \\
\hline Ficus adhatodaefolia Schott ex Spreng. & $\mathrm{Nc}$ & Zooc & & & & & $\mathrm{X}$ \\
\hline Ficus sp. 1 & $\mathrm{Nc}$ & Zooc & & & $\mathrm{X}$ & & \\
\hline Helicostylis tomentosa (Poeep. \& Endl.) Rusby & $\mathrm{Si}$ & Zooc & $\mathrm{X}$ & & & $\mathrm{X}$ & \\
\hline Maclura tinctoria (L.) D. Don ex Steud. & $\mathrm{Nc}$ & Zooc & $\mathrm{X}$ & & & & \\
\hline Sorocea guilleminiana Gaudich. & $\mathrm{Si}$ & Zooc & & & & $\mathrm{X}$ & \\
\hline \multicolumn{8}{|l|}{ MYRSINACEAE } \\
\hline Myrsine coriacea (Sw.) R.Br. ex Roem. \& Schult. & $\mathrm{Si}$ & Zooc & & $\mathrm{X}$ & & & \\
\hline M. umbellata Mart. & St & Zooc & & & & & $X$ \\
\hline \multicolumn{8}{|l|}{ MYRTACEAE } \\
\hline Campomanesia guaviroba (DC.) Kiaersk. & $\mathrm{Si}$ & Zooc & & & & $\mathrm{X}$ & \\
\hline Eugenia stigmatosa DC. & St & Zooc & $\mathrm{X}$ & & & & \\
\hline E. umbrosa Berg. & St & Zooc & $\mathrm{X}$ & & & & \\
\hline Gomidesia spectabilis (DC.) O. Berg & St & Zooc & & & & $\mathrm{X}$ & \\
\hline Myrcia fallax (Rich.) DC. & $\mathrm{Si}$ & Zooc & & & $\mathrm{X}$ & & \\
\hline M. hexasticha Kiaersk. & St & Zooc & $\mathrm{X}$ & & & & \\
\hline M. recurvata $\mathrm{O}$. Berg & St & Zooc & & & & $\mathrm{X}$ & \\
\hline
\end{tabular}


Tabela 1 (continuação)

\begin{tabular}{|c|c|c|c|c|c|c|c|}
\hline \multirow[b]{2}{*}{ Família/Espécie } & \multicolumn{2}{|c|}{ Grupos ecológicos } & \multicolumn{5}{|c|}{ Fragmentos de ocorrência } \\
\hline & $\mathrm{CS}$ & SD & AFT & EST & IMB & AND & STP \\
\hline \multicolumn{8}{|l|}{ MYRTACEAE } \\
\hline Myrcia rostrata DC. & $\mathrm{Si}$ & Zooc & $\mathrm{X}$ & & & $\mathrm{X}$ & \\
\hline Syzygium jambos (L.) Alston* & $\mathrm{Nc}$ & Zooc & $\mathrm{X}$ & & $\mathrm{X}$ & & $\mathrm{X}$ \\
\hline \multicolumn{8}{|l|}{ NYCTAGINACEAE } \\
\hline Guapira nitida (Mart. ex J.A. Schmidt) Lundell & $\mathrm{Si}$ & Zooc & $\mathrm{X}$ & $\mathrm{X}$ & $\mathrm{X}$ & $\mathrm{X}$ & $\mathrm{X}$ \\
\hline G. opposita (Vell.) Reitz & $\mathrm{Si}$ & Zooc & $\mathrm{X}$ & & $\mathrm{X}$ & $\mathrm{X}$ & $\mathrm{X}$ \\
\hline \multicolumn{8}{|l|}{ PHYTOLACCACEAE } \\
\hline Seguieria langsdorffii Moq. & St & Anem & & & & & $\mathrm{X}$ \\
\hline \multicolumn{8}{|l|}{ PIPERACEAE } \\
\hline Piper amalago L. & St & Zooc & & & & & $\mathrm{X}$ \\
\hline P. arboreum Aubl. & St & Zooc & & $\mathrm{X}$ & $\mathrm{X}$ & & \\
\hline \multicolumn{8}{|l|}{ RUBIACEAE } \\
\hline Bathysa mendoncaei K. Schum. & St & Auto & $\mathrm{X}$ & & & $\mathrm{X}$ & $\mathrm{X}$ \\
\hline B. meridionalis L.B. Smith \& Downs & St & Auto & $\mathrm{X}$ & & & & $\mathrm{X}$ \\
\hline Faramea multiflora A. Rich. ex DC. & $\mathrm{Nc}$ & Zooc & & & & & $\mathrm{X}$ \\
\hline Psychotria nuda (Cham. \& Schltdl.) Wawra & $\mathrm{Si}$ & Zooc & & & & $\mathrm{X}$ & \\
\hline P. sessilis (Vell.) Müll. Arg. & $\mathrm{Si}$ & Zooc & $\mathrm{X}$ & & & & \\
\hline P. velloziana Benth. & $\mathrm{Si}$ & Zooc & $\mathrm{X}$ & $\mathrm{X}$ & $\mathrm{X}$ & $\mathrm{X}$ & \\
\hline Randia armata (Sw.) DC. & $\mathrm{Si}$ & Zooc & & & & $\mathrm{X}$ & \\
\hline Rudgea sp. 1 & $\mathrm{Nc}$ & Zooc & $\mathrm{X}$ & & & & \\
\hline Rudgea sp. 2 & $\mathrm{Nc}$ & Zooc & $\mathrm{X}$ & & & $\mathrm{X}$ & \\
\hline Simira sp. 1 & $\mathrm{Nc}$ & Zooc & & & & $\mathrm{X}$ & \\
\hline \multicolumn{8}{|l|}{ RUTACEAE } \\
\hline Zanthoxylum rhoifolium Lam. & $\mathrm{Pi}$ & Zooc & $\mathrm{X}$ & & $\mathrm{X}$ & & \\
\hline Rutaceae sp. 1 & $\mathrm{Nc}$ & $\mathrm{Nc}$ & & & $\mathrm{X}$ & & \\
\hline \multicolumn{8}{|l|}{ SAPINDACEAE } \\
\hline Allophyllus sp. 1 & $\mathrm{Nc}$ & Zooc & & $\mathrm{X}$ & & & \\
\hline Cupania oblongifolia Mart. & $\mathrm{Si}$ & Zooc & $\mathrm{X}$ & $\mathrm{X}$ & $\mathrm{X}$ & $\mathrm{X}$ & $\mathrm{X}$ \\
\hline C. racemosa (Vell.) Radlk. & St & Zooc & & & $\mathrm{X}$ & & \\
\hline Talisia sp.1 & $\mathrm{Nc}$ & Zooc & & & $\mathrm{X}$ & & \\
\hline \multicolumn{8}{|l|}{ SAPOTACEAE } \\
\hline Chrysophyllum flexuosum Mart. & St & Zooc & & $\mathrm{X}$ & $\mathrm{X}$ & $\mathrm{X}$ & $\mathrm{X}$ \\
\hline Chrysophyllum splendens Spreng. & St & Zooc & & & & $\mathrm{X}$ & \\
\hline Ecclinusa ramiflora Mart. & St & Zooc & $\mathrm{X}$ & & & & \\
\hline Micropholis crassipedicellata (Mart. \& Eichler ex Miq.) Pierre & $\mathrm{St}$ & Zooc & & & $\mathrm{X}$ & $\mathrm{X}$ & \\
\hline Picramnia sp. & $\mathrm{Nc}$ & Zooc & & & & & \\
\hline Pouteria bangii (Rusby) T.D. Penn. & St & Zooc & & & & & $\mathrm{X}$ \\
\hline P. caimito (Ruiz \& Pav.) Radlk. & St & Zooc & & & & & $\mathrm{X}$ \\
\hline P. torta (Mart.) Radlk. & St & Zooc & $\mathrm{X}$ & & & $\mathrm{X}$ & $\mathrm{X}$ \\
\hline \multicolumn{8}{|l|}{ SIMAROUBACEAE } \\
\hline Picramnia sp. 1 & $\mathrm{Nc}$ & & $\mathrm{X}$ & & & & \\
\hline \multicolumn{8}{|l|}{ SOLANACEAE } \\
\hline Aureliana fasciculata (Vell.) Sendtn. & $\mathrm{Pi}$ & Zooc & $\mathrm{X}$ & & & & \\
\hline Metternichia princeps Mikan & $\mathrm{Si}$ & Zooc & $\mathrm{X}$ & & & $\mathrm{X}$ & \\
\hline Solanum swartzianum Roem. \& Schult. & $\mathrm{Pi}$ & Zooc & & $\mathrm{X}$ & & & \\
\hline \multicolumn{8}{|l|}{ TILIACEAE } \\
\hline Luehea conwentzii K. Schum. & $\mathrm{Pi}$ & Anem & & & & $\mathrm{X}$ & $\mathrm{X}$ \\
\hline L. divaricata Mart. & $\mathrm{Pi}$ & Anem & $\mathrm{X}$ & $\mathrm{X}$ & $\mathrm{X}$ & $\mathrm{X}$ & $\mathrm{X}$ \\
\hline L. grandiflora Mart. \& Zucc. & $\mathrm{Pi}$ & Anem & $\mathrm{X}$ & $\mathrm{X}$ & $\mathrm{X}$ & $\mathrm{X}$ & $\mathrm{X}$ \\
\hline \multicolumn{8}{|l|}{ VERBENACEAE } \\
\hline Vitex sp. 1 & $\mathrm{Nc}$ & Zooc & $\mathrm{X}$ & & & $\mathrm{X}$ & \\
\hline \multicolumn{8}{|l|}{ INDETERMINADA } \\
\hline Indeterminada sp. 1 & $\mathrm{Nc}$ & $\mathrm{Nc}$ & & & $\mathrm{X}$ & & \\
\hline Indeterminada sp. 2 & $\mathrm{Nc}$ & $\mathrm{Nc}$ & & & $\mathrm{X}$ & & \\
\hline Indeterminada sp. 3 & $\mathrm{Nc}$ & $\mathrm{Nc}$ & & & & & $X$ \\
\hline Indeterminada sp. 4 & $\mathrm{Nc}$ & $\mathrm{Nc}$ & & & & & $\mathrm{X}$ \\
\hline
\end{tabular}


gênero e três $(1,9 \%)$ apenas no nível de família. Quatro morfo-espécies $(2,5 \%)$ permaneceram como indeterminadas.

As famílias que apresentaram maior riqueza de espécies foram Leguminosae (22), Lauraceae (14), Rubiaceae (11), Bignoniaceae (9), Euphorbiaceae (9) e Myrtaceae (9) (Fig. 2A). Em conjunto, estas seis famílias foram responsáveis por $46 \%$ do total de espécies. Em termos de abundância, as famílias que apresentaram o maior número de indivíduos foram Meliaceae (248), Sapindaceae (153), Leguminosae (142), Moraceae (120) e Lauraceae (118) (Fig. 2B) que, juntas, somaram $49 \%$ do total de indivíduos amostrados. Dentre os gêneros, os mais ricos em espécies foram Ocotea, com seis, Nectandra, Miconia e Myrcia com quatro, e Guatteria, Jacaranda, Tabebuia, Psychotria e Luehea com três espécies cada (Tab. 1).

As cinco comunidades analisadas, embora fragmentadas, apresentaram uma alta similaridade de espécies, com valores do índice de Morisita (mod. Horn) variando de 0,36 a 0,79 (Tab. 2). Isto se deve à elevada densidade de algumas espécies comuns aos fragmentos, especialmente das famílias Meliaceae (Guarea guidonia) e Sapindaceae (Cupania
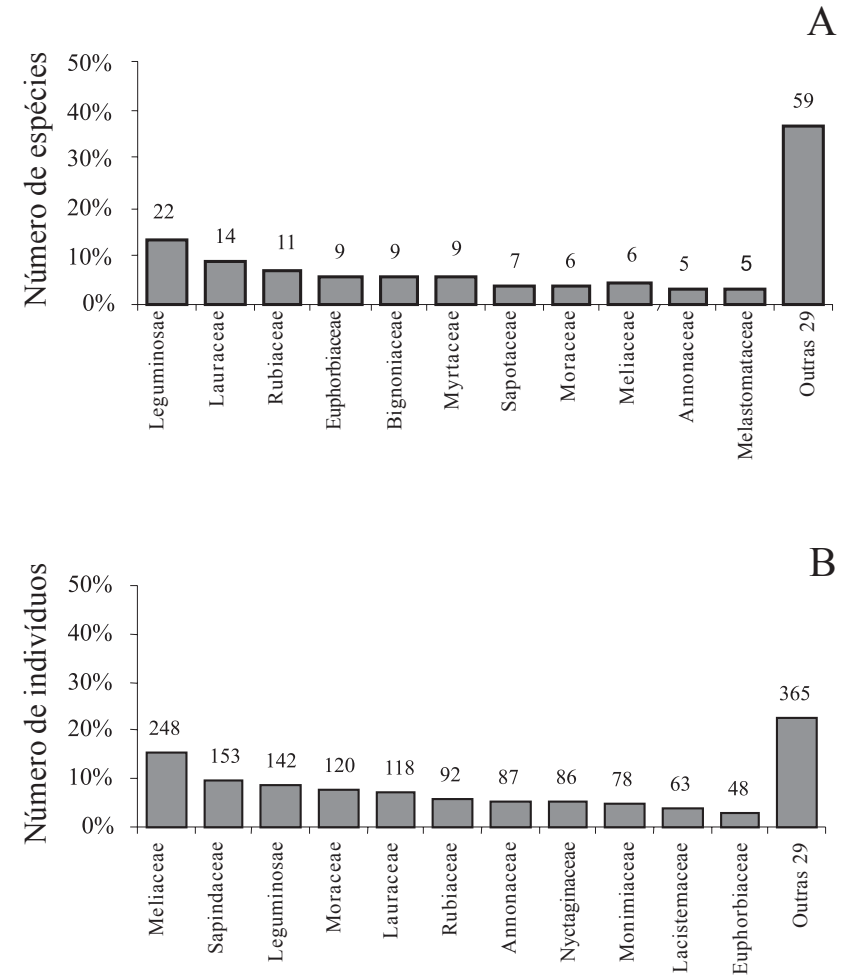

Figura 2. Famílias com maior número de espécies (A) e número de indivíduos (B) na região de Imbaú, Município de Silva Jardim, RJ. Os números sobre as barras correspondem aos valores absolutos.
Tabela 2. Coeficientes de similaridade de espécies de Morisita (mod. Horn) entre os cinco fragmentos de Floresta Ombrófila Densa Submontana estudados na região de Imbaú, Silva Jardim, RJ. Fragmentos: Faz. Afetiva-Jorge (AFT), Faz. Estreito (EST), Faz. Imbaú (IMB), Faz. Andorinhas (AND) e Sítio do Professor (STP).

\begin{tabular}{lccccc}
\hline Fragmentos & AFT & EST & IMB & AND & STP \\
\hline AFT & 1 & 0,403 & 0,420 & 0,678 & 0,364 \\
EST & 0,403 & 1 & 0,698 & 0,365 & 0,789 \\
IMB & 0,420 & 0,698 & 1 & 0,482 & 0,730 \\
AND & 0,678 & 0,365 & 0,482 & 1 & 0,454 \\
STP & 0,364 & 0,789 & 0,730 & 0,454 & 1 \\
\hline
\end{tabular}

oblongifolia). Outras famílias e espécies que contribuíram para a elevada similaridade encontrada foram Annonaceae (Xylopia sericea), Flacourtiaceae (Casearia sylvestris), Lacistemaceae (Lacistema pubescens), Lauraceae (Nectandra oppositifolia e N. puberula), Leguminosae (Plathymenia foliolosa), Monimiaceae (Siparuna guianensis), Nyctaginaceae (Guapira nitida) e Tiliaceae (Luehea divaricata). Em conjunto, estas famílias representaram de 36\% a $66 \%$ do total de indivíduos nos fragmentos.

A elevada riqueza de espécies das famílias Leguminosae e Lauraceae era esperada, visto que tais famílias encontram-se dentre as mais ricas em espécies nas florestas do Estado do Rio de Janeiro (Tab. 3). Além de Leguminosae e Lauraceae, Oliveira Filho \& Fontes (2000), a partir da análise de 125 trechos florestais de Floresta Atlântica, descreveram as famílias Myrtaceae, Rubiaceae, Euphorbiaceae e Melastomataceae, e os gêneros Eugenia, Myrcia, Ocotea e Miconia como os de maior riqueza nas florestas ombrófilas do sudeste brasileiro. Desta forma, os padrões de riqueza encontrados para a região de Imbaú corroboram os encontrados tanto em nível local quanto regional para o bioma Mata Atlântica da região Sudeste.

As famílias que apresentaram alto número de indivíduos no presente estudo, como Leguminosae, Moraceae, Lauraceae, Rubiaceae, Annonaceae, Nyctaginaceae, Monimiaceae e Lacistemaceae, também aparecem entre as de maior densidade em grande parte dos remanescentes de Floresta Ombrófila Densa Submontana secundários da região, ora com maior destaque em um ora em outro levantamento. Entretanto, algumas diferenças relevantes foram observadas nesta comparação. Por exemplo, Meliaceae, que apresentou o maior número de 
Tabela 3. Famílias com maior número de espécies (em \%) nos diferentes trechos de Floresta Atlântica no Estado do Rio de Janeiro. Classificação fitofisionômica segundo Veloso et al. (1991). SO = Floresta Ombrófila Densa Submontana; MO = Floresta Ombrófila Densa Montana; AO = Floresta Ombrófila Densa Alto Montana; BO = Floresta Ombrófila Densa de Terras Baixas; BS = Floresta Semidecídua de Terras Baixas. Famílias: Anacardiaceae (Anac), Annonaceae (Anno), Asteraceae (Aster), Bignoniaceae (Bign), Bombacaceae (Bomb), Chrysobalanaceae (Chrys), Euphorbiaceae (Euph), Lauraceae (Laur), Lecythidaceae (Lecyt), Leguminosae (Legum), Melastomataceae (Melast), Moraceae (Morac), Myrtaceae (Myrt), Nyctaginaceae (Nyct), Rubiaceae (Rubi), Rutaceae (Rutac), Sapotaceae (Sapo), Solanaceae (Solan). *Floresta com perturbação antrópica.

\begin{tabular}{|c|c|c|c|}
\hline Tipo & Localidade & Autor & Famílias mais ricas (\% de sp.) \\
\hline $\mathrm{SO}^{*}$ & Região de Imbaú - Silva Jardim & Este estudo & $\begin{array}{l}\text { Legum }(13,1), \text { Laur }(7,7), \text { Rubi } \\
(6,5), \text { Bign }(5,3), \text { Euph }(5,3)\end{array}$ \\
\hline $\mathrm{SO}^{*}$ & Biovert - Silva Jardim & Borém \& Oliveira Filho (2002) & $\begin{array}{l}\text { Legum }(15,7), \text { Laur }(11,1), \text { Euph } \\
(5,3), \text { Rubi }(5,2), \text { Anno }(3,9)\end{array}$ \\
\hline $\mathrm{SO}^{*}$ & Poço das Antas - Silva Jardim & R.R. Guedes-Bruni (dados não publicados) & $\begin{array}{l}\text { Legum }(15,3), \text { Laur }(10,0), \text { Euph } \\
(7,0), \text { Morac }(6,0), \text { Anno }(5,0)\end{array}$ \\
\hline $\mathrm{SO}^{*}$ & Poço das Antas - Silva Jardim & G.M.S. Neves (dados não publicados) & $\begin{array}{l}\text { Legum }(10,0), \text { Bign }(8,3), \text { Myrt } \\
(8,3), \text { Melast }(6,7), \text { Laur }(5,5)\end{array}$ \\
\hline $\mathrm{SO}^{*}$ & Poço das Antas - Silva Jardim & G.M.S. Neves (dados não publicados) & $\begin{array}{l}\text { Myrt }(12,5), \text { Legum }(10,6), \text { Bign } \\
(5,8) \text {, Euph }(5,8) \text {, Laur }(4,8)\end{array}$ \\
\hline $\mathrm{SO}^{*}$ & Poço das Antas - Silva Jardim & S.V.A. Pessoa (dados não publicados) & $\begin{array}{l}\text { Laur }(10,2), \text { Legum }(10,2), \text { Sapo } \\
(10,2), \text { Euph }(7,0), \text { Myrt }(7,0)\end{array}$ \\
\hline $\mathrm{SO}^{*}$ & Poço das Antas - Silva Jardim & S.V.A. Pessoa (dados não publicados) & $\begin{array}{l}\text { Myrt }(10,1), \text { Laur }(9,8), \text { Anno } \\
(7,0) \text {, Legum }(7,0) \text {, Euph }(6,8)\end{array}$ \\
\hline $\mathrm{SO}^{*}$ & Poço das Antas - Silva Jardim & S.V.A. Pessoa (dados não publicados) & $\begin{array}{l}\text { Myrt }(13,0) \text {, Legum }(10,0), \text { Sapo } \\
(7,0) \text {, Anno }(7,0) \text {, Laur }(7,0)\end{array}$ \\
\hline SO & Imbé $250 \mathrm{~m}$ - Campos dos Goytacazes & Moreno et al. (2003) & $\begin{array}{l}\text { Myrt }(20,0) \text {, Sapot }(18,0) \text {, Legum } \\
(17,0) \text {, Laur }(15,0) \text {, Morac }(12,5)\end{array}$ \\
\hline SO & Imbé $50 \mathrm{~m}$ - Campos dos Goytacazes & Moreno et al. (2003) & $\begin{array}{l}\text { Legum }(17,0) \text {, Laur }(17,0) \text {, Morac } \\
(12,0) \text {, Myrt }(10,0) \text { Sapot }(9,0)\end{array}$ \\
\hline $\mathrm{SO}$ & Paraíso - Guapimirim & Kurtz \& Araújo (2000) & $\begin{array}{l}\text { Myrt }(19,6), \text { Sapot }(10,1), \text { Laur } \\
(10,1) \text {, Rubiac }(6,5), \text { Melast }(4,3)\end{array}$ \\
\hline $\mathrm{SO}$ & União - Rio das Ostras & P.J.F.P. Rodrigues (dados não publicados) & $\begin{array}{l}\text { Myrt }(12,8), \text { Laur }(11,6), \text { Sapo } \\
(11,2), \text { Legum }(9,2) \text { Chrys }(5,2)\end{array}$ \\
\hline $\mathrm{MO}^{*}$ & Macaé de Cima - Nova Friburgo & R.R. Guedes-Bruni (dados não publicados) & $\begin{array}{l}\text { Legum }(14,0), \text { Laur }(13,5), \\
\text { Melast }(10,0), \text { Solan }(6,0), \text { Aster } \\
(6,0)\end{array}$ \\
\hline $\mathrm{MO}$ & Macaé de Cima - Nova Friburgo & R.R. Guedes-Bruni (dados não publicados) & $\begin{array}{l}\text { Melast }(11,6), \text { Laur }(11,3), \text { Myrt } \\
(11,0), \text { Legum }(9,0), \text { Rubiac }(5,1)\end{array}$ \\
\hline BS* & Bom Jesus - Campos dos Goytacazes. & Carvalho et al. (2006) & $\begin{array}{l}\text { Legum }(15,7), \text { Myrt }(11,1), \text { Laur } \\
(6,5), \text { Euph }(5,5) \text {, Anac }(4,6)\end{array}$ \\
\hline BS* & Carvão - São Francisco do Itabapoana & Silva \& Nascimento (2001) & $\begin{array}{l}\text { Legum }(20,5), \text { Myrt }(9,6), \text { Euph } \\
(7,2), \text { Lecyt }(6,0), \text { Rutac }(4,8)\end{array}$ \\
\hline BS & Planície - Búzios & P.R.C. Farág (dados não publicados) & $\begin{array}{l}\text { Myrt. }(22,0), \text { Legum. }(19,0) \text {, } \\
\text { Euph }(8,0) \text {, Nyctag }(4,0), \text { Sapot } \\
(4,0)\end{array}$ \\
\hline BS & Planície-Cabo Frio & H.C. Lima (dados não publicados) & $\begin{array}{l}\text { Legum }(24,7), \text { Myrt }(14,3), \text { Euph } \\
(6,5), \text { Rubi }(5,2), \text { Sapot }(5,2)\end{array}$ \\
\hline BS & Morro - Cabo Frio & H.C. Lima (dados não publicados) & $\begin{array}{l}\text { Legum }(25,2), \text { Rubi }(9,3), \text { Euph } \\
(8,4), \text { Myrt }(7,5), \text { Bign }(2,8)\end{array}$ \\
\hline BO* & Cachoeira Grande - Rio de Janeiro & Peixoto et al. (2004) & $\begin{array}{l}\text { Legum }(19,1), \text { Myrt }(8,8), \text { Euph } \\
(7,4) \text {, Bignon }(4,4), \text { Bombac }(4,4)\end{array}$ \\
\hline $\mathrm{BO}^{*}$ & Poço das Antas - Silva Jardim & R.R. Guedes-Bruni (dados não publicados) & $\begin{array}{l}\text { Legum }(14,5), \text { Euph }(10), \text { Laur } \\
(8,0), \text { Morac }(8,0), \text { Myrt }(8,0)\end{array}$ \\
\hline $\mathrm{AO}$ & Itatiaia & R.R. Guedes-Bruni (dados não publicados) & $\begin{array}{l}\text { Myrt }(19,0), \text { Laur }(12,0), \text { Legum } \\
(10,5), \text { Euph }(6,5), \text { Rubiac }(4,5)\end{array}$ \\
\hline
\end{tabular}


indivíduos neste estudo, não havia sido registrada até o momento dentre as mais abundantes em nenhum dos inventários analisados (Borém \& Oliveira Filho 2002; G.M.S. Neves, dados não publicados; R.R. GuedesBruni, dados não publicados; S.V.A. Pessoa, dados não publicados). Sapindaceae, a segunda mais abundante neste estudo, foi encontrada dentre as mais representativas apenas em trechos florestais na Reserva Biológica de Poço das Antas (G.M.S. Neves, dados não publicados; R.R. Guedes-Bruni, dados não publicados; S.V.A. Pessoa, dados não publicados). Euphorbiaceae, que aparece neste estudo com apenas $3 \%$ dos indivíduos, foi a família que apresentou o maior número de indivíduos em mais da metade dos levantamentos consultados. Num outro extremo, Myrtaceae, que é bastante citada na literatura dentre as mais abundantes, aparece muito pouco representada na região de Imbaú, com apenas $1 \%$ do total de indivíduos. Em conjunto, estes resultados indicam a existência de um padrão de destaque de certas famílias (ex. Euphorbiaceae, Leguminosae, Lauraceae, Moraceae, Myrtaceae e Sapotaceae) na Floresta Ombrófila Densa Submontana da região, o que não exclui a possibilidade de que outras famílias possam vir a se destacar em determinados locais (ex. Meliaceae e Sapindaceae).

Através da comparação da composição florística do dossel (DAP $\geq 10,0 \mathrm{~cm}$ ) da região de Imbaú com a de um trecho de mesma fitofisionomia mais preservado, estudado por P.J.F.P. Rodrigues (dados não publicados) na Reserva Biológica União, foram encontradas diferenças relevantes quanto à riqueza de certas famílias. Nos 1,2 ha amostrados na floresta preservada, foi observada uma predominância das famílias Myrtaceae (32 espécies), Lauraceae (29), Sapotaceae (28), Leguminosae (23), além de Chrysobalanceae, Euphorbiaceae e Moraceae, com 13 espécies cada. Contrapondo-se este resultado ao obtido para o hectare estudado na região de Imbaú, observa-se que as famílias Lauraceae (14), Myrtaceae (9), Sapotaceae (7), Moraceae (5) e principalmente Chrysobalanaceae (2) apresentaram um decréscimo significativo na riqueza de espécies. Tabarelli et al. (1999) também registraram um declínio na riqueza de algumas destas famílias (Lauraceae, Myrtaceae e Sapotaceae) em florestas montanas no Estado de São Paulo, em função da fragmentação e das perturbações antrópicas intrínsecas a este processo. Os autores ainda sugeriram que a redução na riqueza destas famílias, que constituem importantes fontes de alimento para a fauna, possivelmente acarretaria problemas na manutenção de várias espécies frugívoras, principalmente grandes vertebrados, e conseqüentemente alterações na estrutura das comunidades vegetais.

Considerando que a floresta da Reserva Biológica União é bem preservada e apresenta condições próximas ao clímax desta fitofisionomia, confirma-se que a região de Imbaú encontra-se hoje alterada em termos florísticos, em função da fragmentação e distúrbios antrópicos aos quais esteve submetida no passado como extração seletiva de madeira, queimadas e caça. Este fato é corroborado pela distribuição das espécies e seus indivíduos em grupos ecológicos (Fig. 3). Apesar dos resultados indicarem uma maior riqueza das espécies secundárias tardias ( $44 \%$ do total de espécies), observou-se uma predominância dos indivíduos das espécies secundárias iniciais, que somaram aproximadamente $65 \%$ do total de indivíduos. Esta elevada densidade de espécies de grupos sucessionais iniciais vêm sendo apontada como uma característica de matas perturbadas, visto que em florestas tropicais maduras estes grupos tendem a ocorrer em baixas densidades (Hubbel et al. 1999).

Estes resultados mostram que a área ainda não alcançou seu desenvolvimento pleno, ou que existe algum fator impedindo o desenvolvimento de suas comunidades arbóreas. Dentre os prováveis fatores, podem ser destacados o próprio processo de fragmentação florestal ocorrido há várias décadas e as constantes intervenções antrópicas que esta região vêm sofrendo ao longo do tempo (corte seletivo de madeira e queimadas), principalmente por estar localizada em propriedades particulares sem a efetiva proteção. Um outro reflexo destas perturbações é a presença de diversas espécies frutíferas exóticas, como Syzygium jambos (jambo-rosa) e Persea americana (abacate), além de outras observadas fora das parcelas

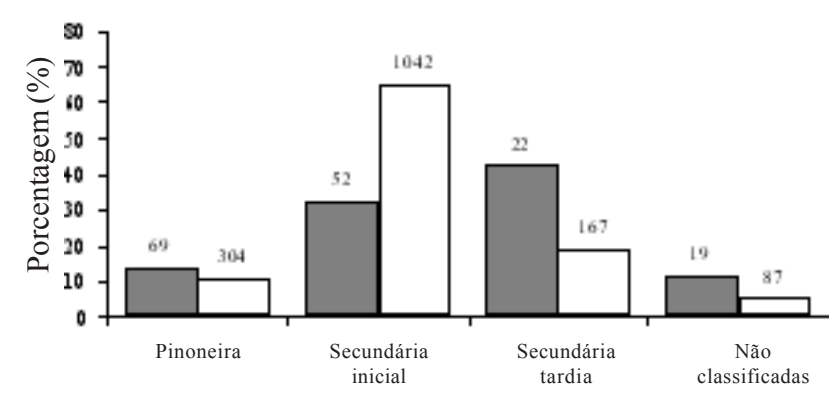

Figura 3. Espécies arbóreas e indivíduos amostrados na região de Imbaú, município de Silva Jardim, RJ, agrupados em grupos ecológicos sucessionais (sensu Gandolfi et al. 1995). Os números sobre as barras correspondem aos valores absolutos. $\square=$ Espécies ( $\mathrm{n}=161) ; \square=$ Indivíduos $(\mathrm{n}=1.598)$. 
amostrais como Mangifera indica (manga), Artocarpus heterophyllus (jaca), Coffea arabica (café) e Psidium guajava (goiaba). Embora estas matas sejam perturbadas, o baixo número de indivíduos de espécies pioneiras, contraposto à elevada densidade de secundárias iniciais, sugere que as mesmas se encontram em estádios intermediários ou avançados de sucessão.

A análise das síndromes de dispersão das espécies amostradas (Tab. 1) indicou que grande parte destas (cerca de 70\%) utiliza-se de animais como agentes de dispersão, padrão já evidenciado em diversos estudos em Floresta Atlântica no sudeste brasileiro (Morellato et al. 1989; Spina et al. 2001; Pessoa 2003; Santos \& Kinoshita 2003). A dispersão de sementes por vertebrados consiste no mecanismo mais importante de disseminação de propágulos em florestas tropicais, onde grandes vertebrados frugívoros, principalmente primatas, exercem um importante papel na reprodução vegetal e regeneração florestal (Howe \& Smallwood 1982). Segundo Ruiz-Miranda et al. (2004), três primatas frugívoros podem ser encontrados na região de Imbaú: o sagüi-de-tufo-branco (Callithrix jaccus), o mico-estrela (Callithrix penicillata) e o mico-leãodourado (Leontopithecus rosalia), o primeiro exótico e invasor e o último constituindo um dos focos da conservação dos remanescentes florestais desta região. Outros frugívoros freqüentemente associados aos remanescentes desta região são pequenos mamíferos (Brito et al. 2004) e principalmente pássaros (J.M.S. Correia, dados não publicados), que exercem importante função na dispersão ao longo das manchas florestais. Neste cenário, a conservação dos remanescentes da região de Imbaú assume grande importância para a manutenção tanto de suas comunidades vegetais quanto da fauna associada.

A região de Imbaú apresentou maior similaridade de espécies com os trechos de Floresta Ombrófila Submontana e em menor grau com os de Floresta Ombrófila de Terras Baixas, Montanas e Altomontanas, e das Florestas Semidecíduas de Terras Baixas do Estado do Rio de Janeiro (Fig. 4). Conforme esperado, os trechos florestais mais similares à região de Imbaú foram aqueles mais próximos geograficamente como os das Reservas Biológicas de Poço das Antas e União, Fazenda Rio Vermelho e Fazenda Biovert, que apresentam mesma fitofisionomia (Floresta Ombrófila Densa Submontana), e situam-se em altitudes, relevos e solos muito semelhantes, além de possuírem históricos de perturbação parecidos.

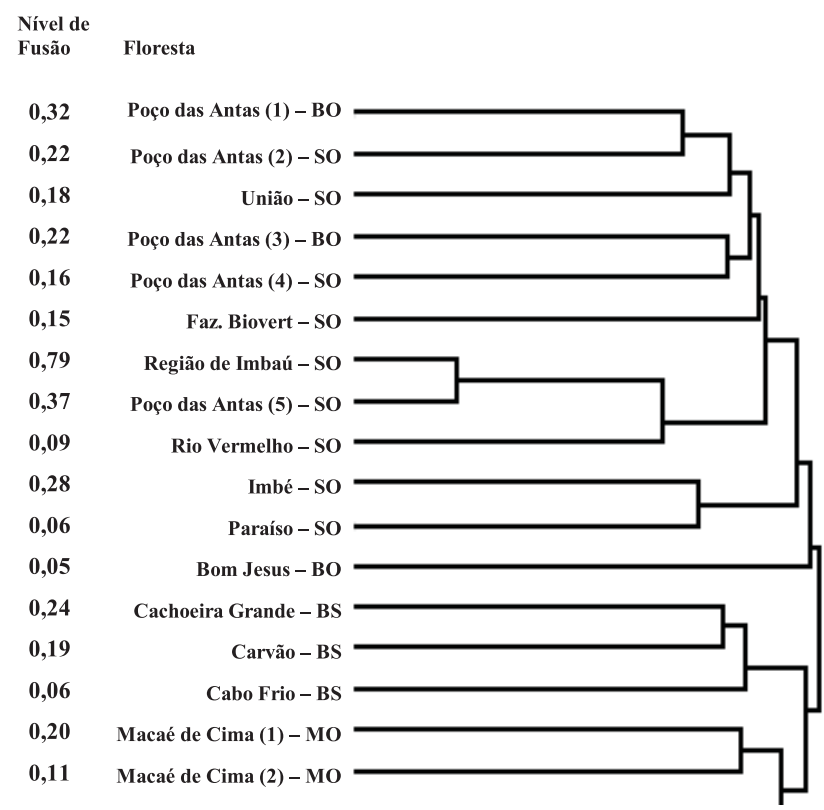

Figura 4. Dendrograma de similaridade de espécies através do índice de Jaccard entre 18 florestas localizadas no Estado do Rio de Janeiro. Método de agrupamento: Média de grupo (UPGMA). Classificação fitofisionômica segundo Veloso et al. (1991). AO Floresta Ombrófila Densa Altomontana; BO - Floresta Ombrófila Densa de Terras Baixas; BS - Floresta Semidecídua de Terras Baixas; MO - Floresta Ombrófila Densa Montana; SO - Floresta Ombrófila Densa Submontana. Referências: Bom Jesus (Carvalho et al. 2006); Cabo Frio (H.C. Lima, dados não publicados); Cachoeira Grande (Peixoto et al. 2004); Carvão (Silva \& Nascimento 2001); Faz. Biovert (Borém \& Oliveira Filho 2002); região de Imbaú (este estudo); Imbé (Moreno et al. 2003); Itatiaia (R.R. Guedes-Bruni, dados não publicados); Macaé de Cima (1 e 2 - R.R. Guedes-Bruni, dados não publicados); Paraíso (R.R. Guedes-Bruni, dados não publicados); Poço das Antas (1 e 2 R.R. Guedes-Bruni, dados não publicados; 3 - F.A. Carvalho \& M.T. Nascimento, dados não publicados; 4 - G.M.S. Neves, dados não publicados; 5 - S.V.A. Pessoa, dados não publicados); Rio Vermelho (Carvalho et al., dados não publicados); União (P.J.F.P. Rodrigues, dados não publicados).

$\mathrm{Na}$ Tabela 4 encontram-se listadas as espécies arbóreas que ocorrem mais freqüentemente nestes trechos de Floresta Ombrófila Densa Submontana, com seus respectivos grupos ecológicos, e que poderiam ser utilizadas em programas de enriquecimento e recuperação de florestas degradadas e ou em desenvolvimento na região de Silva Jardim e seu entorno. Foram incluídas nesta lista as espécies de maior freqüência relativa presentes em pelo menos quatro $(80 \%)$ das cinco regiões florestais analisadas. Das 39 espécies listadas, quatorze foram comuns às cinco regiões: Albizia polycephalla, Brosimum guianense, Cupania oblongifolia, Erythroxylum pulchrum, Guapira nitida, Guarea guidonia, Himatanthus lanceifolius, Mabea fistulifera, 
Siparuna guianensis, Syzygium jambos, Tabebuia chrysotricha, Tabernaemontana laeta, Vernonanthura discolor e Xylopia sericea. As outras 25 espécies que completam a tabela foram encontradas em quatro dos cinco trechos analisados, sendo que todas as espécies relacionadas estiveram presentes na região de Imbaú, a maioria das quais pertencente ao grupo das secundárias iniciais.

A menor similaridade com os demais trechos de Florestas Ombrófilas Densas Submontanas (Imbé e

Tabela 4. Espécies arbóreas mais freqüentes nas regiões de Floresta Ombrófila Densa Submontana da Bacia do Rio São João, RJ. Classificação sucessional (CS) (sensu Gandolfi et al. 1995): pioneira (Pi), secundária inicial ( $\mathrm{Si}$ ), secundária tardia (St) e não classificada $(\mathrm{Nc}) . \mathrm{n}=$ número de trechos florestais analisados.

\begin{tabular}{|c|c|c|c|c|c|c|}
\hline Espécies & $\mathrm{CS}$ & $\begin{array}{l}\operatorname{Imbaú}^{1} \\
(\mathrm{n}=5)\end{array}$ & $\begin{array}{l}\text { Biovert }^{2} \\
(\mathrm{n}=2)\end{array}$ & $\begin{array}{l}\text { União }^{3} \\
(\mathrm{n}=3)\end{array}$ & $\begin{array}{c}\text { Poço Antas }{ }^{4} \\
(n=6)\end{array}$ & $\begin{array}{c}\text { Rio Vermelho }{ }^{5} \\
(\mathrm{n}=1)\end{array}$ \\
\hline Aiouea saligna Meisn. & St & $\mathrm{X}$ & $\mathrm{X}$ & & $\mathrm{X}$ & $\mathrm{X}$ \\
\hline Albizia polycephalla (Benth.) Killip ex Record & $\mathrm{Si}$ & $\mathrm{X}$ & $\mathrm{X}$ & $\mathrm{X}$ & $\mathrm{X}$ & $\mathrm{X}$ \\
\hline Aparisthmium cordatum (A. Juss.) Baill. & $\mathrm{Si}$ & $\mathrm{X}$ & & $\mathrm{X}$ & $\mathrm{X}$ & $\mathrm{X}$ \\
\hline Apuleia leiocarpa Vog. & $\mathrm{Si}$ & $\mathrm{X}$ & & $\mathrm{X}$ & $\mathrm{X}$ & $\mathrm{X}$ \\
\hline Astrocarium aculeatissimum (Schott) Burret & $\mathrm{Pi}$ & $\mathrm{X}$ & & $\mathrm{X}$ & $\mathrm{X}$ & $\mathrm{X}$ \\
\hline Bathysa mendoncaei K. Schum. & St & $X$ & & $\mathrm{X}$ & $\mathrm{X}$ & $\mathrm{X}$ \\
\hline Brosimum guianense (Aubl.) Huber & $\mathrm{Si}$ & $\mathrm{X}$ & $\mathrm{X}$ & $\mathrm{X}$ & $\mathrm{X}$ & $\mathrm{X}$ \\
\hline Casearia arborea (Rich.) Urb. & $\mathrm{Si}$ & $\mathrm{X}$ & & $\mathrm{X}$ & $\mathrm{X}$ & $\mathrm{X}$ \\
\hline C. sylvestris Sw. & $\mathrm{Si}$ & $\mathrm{X}$ & $\mathrm{X}$ & & $\mathrm{X}$ & $\mathrm{X}$ \\
\hline Ceiba crispiflora (Humb., Bompl. \& Kunth.) P. Ravenna & St & $\mathrm{X}$ & $\mathrm{X}$ & $\mathrm{X}$ & $\mathrm{X}$ & \\
\hline Chrysophyllum flexuosum Mart. & St & $\mathrm{X}$ & & $\mathrm{X}$ & $\mathrm{X}$ & $\mathrm{X}$ \\
\hline Cupania oblongifolia Mart. & $\mathrm{Si}$ & $\mathrm{X}$ & $\mathrm{X}$ & $\mathrm{X}$ & $\mathrm{X}$ & $X$ \\
\hline Cybistax antisyphilitica Mart. & $\mathrm{Pi}$ & $\mathrm{X}$ & $\mathrm{X}$ & & $\mathrm{X}$ & $\mathrm{X}$ \\
\hline Dalbergia nigra (Vell.) Allemão ex Benth. & St & $\mathrm{X}$ & $\mathrm{X}$ & & $\mathrm{X}$ & $\mathrm{X}$ \\
\hline Erythroxylum pulchrum St. Hilaire & St & $\mathrm{X}$ & $\mathrm{X}$ & $\mathrm{X}$ & $\mathrm{X}$ & $\mathrm{X}$ \\
\hline Guapira nitida (Mart. ex J.A. Schmidt) Lundell. & $\mathrm{Si}$ & $X$ & $\mathrm{X}$ & $\mathrm{X}$ & $\mathrm{X}$ & $\mathrm{X}$ \\
\hline Guarea guidonea (L.) Sleumer & $\mathrm{Si}$ & $\mathrm{X}$ & $\mathrm{X}$ & $\mathrm{X}$ & $\mathrm{X}$ & $\mathrm{X}$ \\
\hline Guatteria candolleana Schlecht. & $\mathrm{Si}$ & $\mathrm{X}$ & $\mathrm{X}$ & & $\mathrm{X}$ & $\mathrm{X}$ \\
\hline G. xylopioides R.E.Fr. & $\mathrm{Si}$ & $\mathrm{X}$ & $\mathrm{X}$ & $\mathrm{X}$ & $\mathrm{X}$ & \\
\hline Himatanthus lanceifolius (Muell. Arg.) R.E. Woodson & St & $\mathrm{X}$ & $\mathrm{X}$ & $\mathrm{X}$ & $\mathrm{X}$ & $\mathrm{X}$ \\
\hline Jacaranda bracteata Bur. \& K.Schum. & $\mathrm{Si}$ & $\mathrm{X}$ & & $\mathrm{X}$ & $\mathrm{X}$ & $\mathrm{X}$ \\
\hline Lacistema pubescens Mart. & $\mathrm{Si}$ & $\mathrm{X}$ & & $\mathrm{X}$ & $\mathrm{X}$ & $\mathrm{X}$ \\
\hline Luehea conwentzii K. Schum. & $\mathrm{Pi}$ & $\mathrm{X}$ & $\mathrm{X}$ & $\mathrm{X}$ & $\mathrm{X}$ & \\
\hline L. divaricata Mart. & $\mathrm{Pi}$ & $\mathrm{X}$ & & $\mathrm{X}$ & $\mathrm{X}$ & $\mathrm{X}$ \\
\hline Mabea fistulifera Mart. & $\mathrm{Pi}$ & $\mathrm{X}$ & $\mathrm{X}$ & $\mathrm{X}$ & $\mathrm{X}$ & $\mathrm{X}$ \\
\hline Machaerium brasiliensis Vog. & $\mathrm{Si}$ & $\mathrm{X}$ & & $\mathrm{X}$ & $\mathrm{X}$ & $\mathrm{X}$ \\
\hline Nectandra oppositifolia Nees. & $\mathrm{Si}$ & $\mathrm{X}$ & & $\mathrm{X}$ & $\mathrm{X}$ & $\mathrm{X}$ \\
\hline N. puberula (Schott) Nees & $\mathrm{Si}$ & $\mathrm{X}$ & $\mathrm{X}$ & $\mathrm{X}$ & $\mathrm{X}$ & \\
\hline Ocotea aniboides $\mathrm{Mez}$ & St & $\mathrm{X}$ & $\mathrm{X}$ & $\mathrm{X}$ & $\mathrm{X}$ & \\
\hline Pera glabrata (Schott) Poepp. ex Baill. & $\mathrm{Si}$ & $\mathrm{X}$ & & $\mathrm{X}$ & $\mathrm{X}$ & $\mathrm{X}$ \\
\hline P. leandri Baill. & St & $\mathrm{X}$ & & $\mathrm{X}$ & $\mathrm{X}$ & $\mathrm{X}$ \\
\hline Plathymenia foliolosa Benth. & St & $\mathrm{X}$ & & $\mathrm{X}$ & $\mathrm{X}$ & $\mathrm{X}$ \\
\hline Platycyanus regnellii Benth. & St & $\mathrm{X}$ & $\mathrm{X}$ & $\mathrm{X}$ & $\mathrm{X}$ & \\
\hline Siparuna guianensis (Tul.) A. DC. & $\mathrm{Si}$ & $\mathrm{X}$ & $\mathrm{X}$ & $\mathrm{X}$ & $\mathrm{X}$ & $\mathrm{X}$ \\
\hline Swartzia simplex Spreng. & $\mathrm{St}$ & $\mathrm{X}$ & $\mathrm{X}$ & & $\mathrm{X}$ & $\mathrm{X}$ \\
\hline Syzygium jambos (L.) Alston* & $\mathrm{Nc}$ & $\mathrm{X}$ & $\mathrm{X}$ & $\mathrm{X}$ & $\mathrm{X}$ & $\mathrm{X}$ \\
\hline Tabebuia chrysotricha (Mart. ex DC.) Standley & $\mathrm{St}$ & $\mathrm{X}$ & $\mathrm{X}$ & $\mathrm{X}$ & $\mathrm{X}$ & $\mathrm{X}$ \\
\hline Tabernaemontana laeta Mart. & St & $\mathrm{X}$ & $\mathrm{X}$ & $\mathrm{X}$ & $\mathrm{X}$ & $\mathrm{X}$ \\
\hline Tapirira guianensis Aubl. & $\mathrm{Si}$ & $\mathrm{X}$ & & $\mathrm{X}$ & $\mathrm{X}$ & $\mathrm{X}$ \\
\hline Tovomitopsis paniculata Planch. \& Triana & $\mathrm{Nc}$ & $\mathrm{X}$ & $\mathrm{X}$ & $\mathrm{X}$ & $\mathrm{X}$ & \\
\hline Vernonanthura discolor (Less.) H. Rob. & $\mathrm{Pi}$ & $\mathrm{X}$ & $\mathrm{X}$ & $\mathrm{X}$ & $\mathrm{X}$ & $\mathrm{X}$ \\
\hline Vismia guianensis (Aubl.) Choisy & $\mathrm{Si}$ & $\mathrm{X}$ & & $\mathrm{X}$ & $\mathrm{X}$ & $\mathrm{X}$ \\
\hline Xylopia sericea A. St.-Hil. & $\mathrm{Pi}$ & $\mathrm{X}$ & $\mathrm{X}$ & $\mathrm{X}$ & $\mathrm{X}$ & $\mathrm{X}$ \\
\hline
\end{tabular}

${ }^{1}$ Região de Imbaú (este estudo); ${ }^{2}$ Faz. Biovert (Borém \& Oliveira Filho 2002); ${ }^{3}$ Reserva Biológica União (P.J.F.P. Rodrigues, dados não publicados); ${ }^{4}$ Reserva Biológica de Poço das Antas (R.R. Guedes-Bruni, dados não publicados; G.M.S. Neves, dados não publicados; S.V.A. Pessoa, dados não publicados); ${ }^{5}$ Faz. Rio Vermelho (F.A. Carvalho \& M.T. Nascimento, dados não publicados). *Espécie exótica. 
Paraíso) foi atribuída a dois fatores: à maior distância geográfica em relação à região de Imbaú e ao maior grau de conservação destas florestas. Dentre as espécies comuns a estas florestas estão, além de várias das mencionadas anteriormente, algumas características de estádios sucessionais mais avançados, a exemplo de Cariniana legalis, Chrysophyllum flexuosum, Ecclinusa ramiflora, Melanoxylon brauna, Mycropholis crassipedicelata, Pouteria caimito e Pouteria torta.

Com relação aos demais trechos comparados (Florestas Semidecíduas de Terras Baixas e Florestas Ombrófilas Densas Montanas e Altomontanas), a baixa similaridade de espécies encontrada era esperada por se tratarem de fitofisionomias distintas, estabelecidas sob diferentes condições de altitude e clima, além de estarem mais distantes geograficamente da região de Imbaú. Apesar dos poucos estudos florísticos comparativos no Estado do Rio de Janeiro, a maior similaridade de espécies entre trechos de mesma fitofisionomia já havia sido reportada por Moreno et al. (2003) e Peixoto et al. (2004), em função das variações climáticas e altitudinais que ocorrem ao longo do gradiente mar-interior.

Desta forma, os padrões de similaridade observados neste estudo corroboram os descritos para as florestas do bioma Mata Atlântica, onde a diferenciação taxonômica encontra-se fortemente relacionada ao regime de chuvas (embora as transições possam ser graduais) e à altitude e suas correspondentes variações de temperatura (Torres et al. 1997; Oliveira Filho \& Fontes 2000; Scudeller et al. 2001). Em síntese, os resultados aqui apresentados indicam que, além dos fatores geográficos e climáticos, a perturbação da área é uma outra informação que deve ser levada em consideração nas comparações florísticas, o que, entretanto, vêm sendo pouco aplicada nos estudos comparativos da flora brasileira.

$\mathrm{Na}$ atual paisagem fragmentada da região CentroNorte Fluminense, a falta de opções de tamanhos e formatos desejáveis para a implementação de Unidades de Conservação torna os pequenos remanescentes as únicas alternativas para a preservação da biodiversidade local. Os resultados aqui apresentados mostram que, apesar de secundários, os remanescentes florestais da região de Imbaú assumem grande relevância na manutenção da fauna e flora local, servindo, por exemplo, como áreas de conectividade e facilitação ao fluxo de dispersores, e como bancos genéticos ex situ de espécies vegetais importantes para programas de manejo e restauração florestal. No entanto, conforme sugerido por Carvalho et al. (2004), a efetiva conservação destas florestas só será possível quando aliada a estudos preliminares e a um manejo florestal bem planejado e contextualizado na paisagem regional, com o enriquecimento florestal adequado à manutenção da fauna associada.

\section{Agradecimentos}

Os autores agradecem aos pesquisadores Ary T. Oliveira Filho, Dorothy S.D. Araujo, Dora M. Villela, José R.R. Pinto e Pablo J.F.P. Rodrigues, pela revisão e contribuições ao manuscrito; ao técnico Heuzenil S. Cordeiro, pelo auxílio nos trabalhos de campo; ao Márcio Schmidt, pela confecção do mapa; aos pesquisadores do Jardim Botânico do Rio de Janeiro, Alexandre Quinet, Carine Pinto-Quinet, Cláudia M. Vieira, Cyl Farney, Massimo G. Bovini e Haroldo C. de Lima, pelo auxílio na identificação do material botânico; ao FNMA/MMA, pelo apoio financeiro; ao Laboratório de Ciências Ambientais da Universidade Estadual do Norte Fluminense, Associação Mico Leão Dourado e IBAMA, pelo apoio logístico; a CAPES, pela concessão da bolsa de mestrado ao primeiro autor.

\section{Referências bibliográficas}

Ayres, J.M.; Fonseca, G.A.B.; Rylands, A.B.; Queiroz, H.L.; Pinto, L.P.; Masterson, D. \& Cavalcanti, R.B. 2005. Os corredores ecológicos das florestas tropicais do Brasil. Belém, Sociedade Civil Mamirauá.

Borém, R.T. \& Oliveira Filho, A.T. 2002. Fitossociologia do estrato arbóreo em uma topossequência alterada de Mata Atlântica, no município de Silva Jardim-RJ, Brasil. Revista Árvore 26(6): 727-742.

Brito, D.; Oliveira, L.C. \& Mello, M.A.R. 2004. An overview of mammalian conservation at Poço das Antas Biological Reserve, southeastern Brazil. Journal of Nature Conservation 12: 219-228.

Brower, J.E. \& Zar, J.H. 1984. Field and laboratory methods for general ecology. $2^{\text {nd }}$ ed. Iowa, W.C. Brown Company Publishers.

Câmara, I.G. 1983. Tropical moist forest conservation in Brazil. Pp. 413-421. In: S.L. Sutton; T.C. Whitmore \& A.C. Chadwick (eds.). Tropical Rain Forest: ecology and management. Oxford, Blackwell Scientific Publications.

Carvalho, F.A.; Nascimento, M.T.; Oliveira, P.P.; Rambaldi, D.M. \& Fernandes, R.V. 2004. A importância dos remanescentes florestais da Mata Atlântica de baixada costeira fluminense para a conservação da biodiversidade na APA da Bacia do Rio São João/MicoLeão-Dourado/IBAMA - RJ. Pp. 106-113. In: Anais do IV Congresso Brasileiro de Unidades de Conservação, vol. 1. Curitiba, Fundação $O$ Boticário de Proteção à Natureza: Rede Nacional Pró Unidades de Conservação. 
Carvalho, F.A.; Braga, J.M.A.; Gomes, J.M.L.; Souza, J.S. \& Nascimento, M.T. 2006. Comunidade arbórea de uma floresta de baixada aluvial no município de Campos dos Goytacazes, RJ. CERNE 12(2): 157-166.

Causton, D.R. 1988. An introduction to vegetation analysis, principles, practice and interpretation. London, Unwin Hyman.

Cronquist, A. 1981. An integrated system of classification of flowering plants. New York, Columbia University Press.

Dean, W. 1996. A ferro e fogo: a história e a devastação da Mata Atlântica brasileira. São Paulo, Companhia das Letras.

Felfili, J.M. \& Rezende, R.P. 2003. Conceitos e Métodos em Fitossociologia. Comunicações Técnicas Florestais. v.5, n.1. Brasília, Universidade de Brasília, Departamento de Engenharia Florestal.

Fundação SOS Mata Atlântica. 2002. Atlas da evolução dos remanescentes florestais e ecossistemas associados no domínio da Mata Atlântica no período 1995-2000. São Paulo, SOS Mata Atlântica/INPE/ISA.

Gandolfi, S.; Leitão Filho, H.F. \& Bezerra, C.L.F. 1995. Levantamento florístico e caráter sucessional das espécies arbustivo-arbóreas de uma floresta mesófila semidecídua no município de Guarulhos, SP. Revista Brasileira de Biologia 55(4): 753-767.

Howe, H.F. \& Smallwood, J. 1982. Ecology of seed dispersal. Annual Review of Ecology and Systematics 13: 201-228.

Hubbel, S.P.; Foster, R.B.; O’Brien, S.T.; Harms, K.E.; Condit, R.; Wechsler, B.; Wright, S.J. \& Lao, S.L. 1999. Light gaps disturbance, recruitment limitations and tree diversity in a neotropical forest. Science 283: 554-557.

IBDF. 1981. Plano de manejo da Reserva Biológica de Poço das Antas. Documento técnico n. 10. Brasília, Ministério da Agricultura.

IBGE. 2004. Mapa de solos do Brasil. Disponível em : http:/ /www.ibge.gov.br. (Acesso em 18/12/2004).

Koeppen, W. 1948. Climatologia. Mexico, Ed. Fondo de la Cultura Economica.

Kurtz, B.C. \& Araújo, D.S.D. 2000. Composição florística e estrutura do componente arbóreo de um trecho de Mata Atlântica na Estação Ecológica Estadual do Paraíso, Cachoeira de Macacú, Rio de Janeiro, Brasil. Rodriguésia 51: 69-112.

MMA. 2000. Avaliação e ações prioritárias para a conservação da biodiversidade da Mata Atlântica e Campos Sulinos. Brasília, MMA/SBF.

Morellato, L.P.C.; Rodrigues, R.R.; Leitão Filho, H.F. \& Joly, C.A. 1989. Estudo comparativo da fenologia de espécies arbóreas de floresta de altitude e floresta mesófila semidecídua na Serra do Japi, Jundiaí, São Paulo. Revista Brasileira de Botânica 12(1): 85-91.

Moreno, M.R.; Nascimento, M.T. \& Kurtz, B.C. 2003. Estrutura e composição florística do estrato arbóreo em duas zonas altitudinais na Mata Atlântica de encosta da região do Imbé, RJ. Acta Botanica Brasilica 17(3): 371-386.

Myers, N.; Mittermeier, R.A.; Mittermeier, C.G.; Fonseca, G.A.B. \& Kent, J. 2000. Biodiversity hotsposts for conservation priorities. Nature 403: 845-853.
Oliveira Filho, A.T. \& Fontes, M.A.L. 2000. Patterns of floristic differentiation among Atlantic Forests in Southeastern Brazil and the influence of climate. Biotropica 32(4b): 793-810.

Peixoto, G.L.; Martins, S.V.; Silva, A.F. \& Silva, E. 2004. Composição florística do componente arbóreo de um trecho de Floresta Atlântica na Área de Proteção Ambiental da Serra da Capoeira Grande, Rio de Janeiro, RJ, Brasil. Acta Botanica Brasilica 18(1): 151-160

Primo, P.B. \& Völker, C.M. 2003. Bacias hidrográficas dos rios São João e das Ostras - águas, terras e conservação ambiental. Rio de Janeiro, CILSJ.

Programa Mata Atlântica. 1996. Relatório Técnico-Científico 1993-1996. Rio de Janeiro, Jardim Botânico do Rio de Janeiro/IBAMA.

Rambaldi, D.M. \& Oliveira, D.A.S. (orgs.). 2003. Fragmentação de ecossistemas: causas, efeitos sobre a biodiversidade e recomendações de políticas públicas. Brasília, MMA/SBF.

Royal Botanical Garden of Kew. 1993. Index Kewensis on compact disc - manual. Oxford, Oxford University Press.

Ruiz-Miranda, C.R.; Morais, M.M.; Beck, B.B. \& Verona, C.E. 2004. O impacto de espécies invasoras em comunidades naturais - o caso do mico-estrela vs. micoleão-dourado. Pp. 64-73. In: Anais do IV Congresso Brasileiro de Unidades de Conservação. v. 2. Curitiba, Fundação O Boticário de Proteção à Natureza: Rede Nacional Pró Unidades de Conservação.

Santos, K. \& Kinoshita, L.S. 2003. Flora arbustivo-arbórea do fragmento de floresta estacional semidecidual do Ribeirão Cachoeira, Município de Campinas, SP. Acta Botanica Brasilica 17(3): 325-341.

Scudeller, V.V.; Martins, F.R. \& Sheperd, G.J. 2001. Distribution and abundance of arboreal species in the Atlantic ombrophilous dense forest in Southeastern Brazil. Plant Ecology 152: 185-199.

Silva, G.C. \& Nascimento, M.T. 2001. Fitossociologia de um remanescente de mata sobre tabuleiros no norte do Estado do Rio de Janeiro (Mata do Carvão). Revista Brasileira de Botânica 24(1): 51-62.

Spina, A.P.; Ferreira, W.M. \& Leitão Filho, H.F. 2001. Floração, frutificação e síndromes de dispersão de uma comunidade de floresta de brejo na região de Campinas (SP). Acta Botanica Brasilica 15(3): 47-60.

Tabarelli, M.; Mantovani, W. \& Peres, C.A. 1999. Effects of habitat fragmentation on plant guild structure in the montane Atlantic forest of Southeastern Brazil. Biological Conservation 91: 119-127.

Tabarelli, M.; Pinto, L.P.; Silva, J.MC.; Hirota, M.M. \& Bedê, L.C. 2005. Desafios e oportunidades para a conservação da biodiversidade na Mata Atlântica brasileira. Megadiversidade 1(1): 132-138.

Torres, R.B.; Martins, F.R. \& Kinoshita, L.S. 1997. Climate, soil and tree flora relationships in forest in the State of São Paulo, southeastern Brazil. Revista Brasileira de Botânica 20(1): 41-49.

Van der Pijl, L. 1982. Principles of dispersal in higher plants. Berlin, Springer-Verlag.

Veloso, H.P.; Rangel Filho, A.L.R. \& Lima, J.C.A. 1991. Classificação da vegetação brasileira adaptada a um sistema universal. Rio de Janeiro, IBGE. 